\title{
Análise dos índices de adiposidade e de aptidão física em crianças pré-púberes
}

\author{
Fabrício B. Alves \\ Anabelle M. Barbosa \\ Wagner de Campos \\ Ricardo W. Coelho \\ Sérgio G. da Silva
}

\author{
Centro de Pesquisa em Esporte e Exercício \\ Universidade Federal do Paraná \\ Brasil
}

\section{RESUMO}

O objectivo deste estudo foi avaliar indicadores de aptidão física em crianças pré-púberes dos dois sexos com idades compreendidas entre os 8-11 anos e em cada sexo (32 meninos e 35 meninas) as relações existentes entre diferentes indicadores de adiposidade. Os estágios de maturação sexual foram considerados e determinados por método de auto-avaliação modificado. Os indicadores de adiposidade considerados foram o percentual de gordura a partir de medidas das dobras cutâneas do tríceps e subescapular) e os perímetros da cintura e quadril. Para a avaliação da aptidão física recorreu-se aos testes de sentar e alcançar, de preensão manual e ao teste de $20 \mathrm{~m}$ de corrida em vai-vém para estimação do $\mathrm{VO}_{2 \max }$. Nos dois sexos, registaram-se correlações significativas $(\mathrm{p}<0,05)$ entre a percentagem de gordura, o IMC, e perímetros da cintura e quadril. O IMC foi melhor predito $(89,7 \%)$ pelo percentual de gordura, massa corporal magra e idade. A massa corporal magra foi diferenciada entre sexos $(p<0,05)$. Os meninos apresentaram nível de flexibilidade discretamente menos elevado ( $p>0,05)$; melhor nível de força de preensão manual e $\mathrm{VO}_{2}$ max do que as meninas $(\mathrm{p}<0,05)$. Recomenda-se que o estágio maturacional por sexo seja considerado na análise dos indicadores de adiposidade e de aptidão física em crianças.

\begin{abstract}
Analysis of adiposity and physical fitness indexes in prepubertal children

The purpose of this study was the to assess physical fitness in prepubertal children aged 8-11 years old and, and by gender (32 boys and 35 girls) to analyze the relationships between adiposity indexes. The drawings of self-assessment modified by Faulkner were used to assess the stage of sexual maturation. Fat percentage was calculated by Boileau et al. equations, waist and hip perimeters were collected by Callaway et al. standardization. Sit-and-reach test and hand grip tests were performed according to Johnson and Nelson and Safrit. $V_{2 \max }$ estimation was had using 20-m Shuttle Run test developed by Léger et al.. As a result, there were significant correlations $(p<0.05)$ among BMI, fat percentage and waist and hip perimeter. BMI awareness was best predicted (89.7\%) by fat percentage, lean body mass and age. The obtained lean body mass was different between sexes $(p<0.05)$. Scientific outcomes for boys presented lower level of flexibility $(p>0.05)$; higher levels of handgrip strength and $\mathrm{VO}_{2 \max }(p<0.05)$. Sharply, maturational stage and sex should be considered in the analysis of adiposity and fitness in children.
\end{abstract}

Key-words: adiposity, fitness, prepubertal

Palavras-chave: adiposidade, aptidão física, pré-púberes. 


\section{INTRODUÇÃ̃o}

O conceito de aptidão física relacionado à saúde é operacionalizado por itens como a resistência cardiorespiratória, força muscular, flexibilidade e composição corporal. A aptidão física na infância é influenciada por factores como a idade, sexo, composição corporal, status de maturidade biológica, entre outros ${ }^{(14)}$.

O sobrepeso e a obesidade infantil são considerados mundialmente como uma epidemia e problema de saúde pública e talvez as morbilidades associadas mais importantes a curto prazo sejam de natureza psicossocial, incluindo, a marginalização social, a diminuição da auto-estima e também a diminuição da qualidade de vida (29).

A avaliação do início e progressão da maturação sexual é importante para a interpretação clínica do status de crescimento e metabólico(24), principalmente, devido ao facto de em jovens se observar elevada variação maturacional em idades cronológicas semelhantes e também por se ter demonstrado que a maturação sexual está associada ao sobrepeso e obesidade (28).

A obesidade em crianças está associada e tende a agregar-se a factores de risco para a saúde como a hipertensão, a hipercolesterolemia, a hipertrigliceridemia, o aumento do colesterol-(LDL) ${ }^{(1)}$, e do colesterol-(VLDL), à diminuição do colesterol HDL, à hiperinsulinemia, à resistência a insulina e diabetes tipo 2 e ainda ao início e progressão de lesões ateroscleróticas. Devido ao facto de a obesidade na infância predizer a obesidade na idade adulta jovem e de isso, em conjugação com a presença de outros factores de risco predizer o aumento do risco de mortalidade e morbilidade é que a abordagem da obesidade deve estar inclusa nos estudos que tratam sobre a infância(30).

O IMC é reconhecidamente um preditor da morbidade e da mortalidade que ocorrem devido a doenças crónicas nas quais se incluem a diabetes tipo 2, as doenças cardiovasculares e os acidentes cerebrovasculares. Porém, existem evidências de a associação entre o IMC e a medida do perímetro da cintura ser um melhor preditor dos riscos para a saúde do que o valor do IMC considerado isoladamente(15). Em crianças, a distribuição centralizada da massa gorda, representada pelo perímetro da cintura, representa um risco mais elevado para complicações metabóli- cas, ou seja, para concentrações plasmáticas mais elevadas do colesterol LDL e triglicerídeos e para o de níveis menos elevados de colesterol HDL e insulina basal(20). Porém, a associação entre o perímetro do quadril e o risco para a saúde é negativa e isto pode ser justificado pela presença de massa muscular mais elevada em regiões não abdominais. A massa corporal magra apresenta relação negativa com índices de mortalidade o que sugere um efeito protector contra riscos relatados à saúde(15).

Este estudo de avaliação dos componentes de aptidão física relacionada à saúde teve por objectivo analisar em crianças pré-púberes entre sexos os indicadores de aptidão física e por sexo as relações existentes entre os índices de adiposidade corporal.

\section{METODOLOGIA}

\section{População e Amostra}

Foram avaliados 32 meninos e 35 meninas pré-púberes na faixa etária entre 8 a 11 anos matriculados em escolas da rede pública de Curitiba-PR. Todas as pessoas que participaram do estudo foram autorizadas pelo seu pai ou responsável e estavam cientes dos procedimentos que seriam realizados e das implicações do estudo. O estudo foi aprovado pelo Comité de Ética em Pesquisa envolvendo seres humanos da Universidade Federal do Paraná.

\section{Procedimentos}

As avaliações decorreram em 3 dias diferentes. No primeiro dia, os potenciais participantes foram informados dos objectivos e procedimentos do estudo e foi-lhes entregue uma carta dirigida aos pais ou responsáveis com o mesmo tipo de informação e que incluía, também, o termo de autorização que deveria ser por eles assinado caso anuíssem a que os seus educandos participassem no estudo. No segundo dia foram mensuradas a massa corporal, estatura, altura tronco-cefálica, altura trocantérica, perímetro da cintura e do quadril, dobras cutâneas subescapular e tricipital e a força de preensão manual. A auto-avaliação da maturação sexual realizada em forma de entrevista, a avaliação da flexibilidade e o teste para a estimação do $\mathrm{VO}_{2 \max }$ ocorreram no terceiro dia das avaliações.

Para a auto-avaliação da maturação sexual foram usados os desenhos do protocolo modificado por 
Faulkner(10); para os meninos os desenhos representavam o desenvolvimento do aparelho reprodutor masculino e para as meninas o desenvolvimento das mamas. Os avaliadores que realizaram as entrevistas eram indivíduos experimentados neste método e nos procedimentos de entrevista, tendo tido o cuidado de explicaram os desenhos às crianças para evitar problemas de compreensão e entendimento daquilo que elas observavam.

O percentual de gordura foi calculado pela equação de Boileau et al.(3), que utiliza as dobras cutâneas tricipital (TR, mm) e subescapular (SE, mm). As equações utilizadas foram:

$$
\begin{gathered}
\text { meninos } \\
\text { Percentual de gordura }= \\
(1,35 \times(\mathrm{TR}+\mathrm{SE}))-\left(0,012 \times(\mathrm{TR}+\mathrm{SE})^{2}\right)-4,4 \\
\text { meninas } \\
\text { Percentual de gordura }= \\
(1,35 \times(\mathrm{TR}+\mathrm{SE}))-\left(0,012 \times(\mathrm{TR}+\mathrm{SE})^{2}\right)-2,4 .
\end{gathered}
$$

As medidas da massa corporal e estatura foram colectadas pelos procedimentos sugeridos por Gordon et al.(11), sendo os instrumentos utilizados a balança com escala de 100 gramas e o estadiômetro com escala em centímetros $(\mathrm{cm})$. Para a medição das dobras cutâneas utilizou-se um plicômetro ( $\mathrm{mm}$ ) de acordo com os procedimentos descritos por Harrison et al.(12). Para a determinação das medidas dos perímetros da cintura e do quadril utilizou-se uma fita antropométrica com escala em milimetros ( $\mathrm{mm}$ ) e seguiram-se procedimentos padronizados sugeridos por Callaway et al.(5). As mensurações da flexibilidade e da força muscular foram efectuadas segundo as recomendações de Johnson e Nelson(16) e Safrit(21) e os instrumentos utilizados foram, respectivamente, a caixa de madeira construída para o teste de sentar e alcançar com escala de $0,5 \mathrm{~cm}$ e o centímetro 23 coincidindo com a região plantar dos pés, e um dinamômetro manual (com escala em $\mathrm{Kg}$ ). O consumo máximo de oxigênio foi estimado a partir da performance obtida em teste de corrida vai-vem (20-m Shuttle Run Test) de acordo com os procedimentos e equação indicados por Léger et al.(17). Para esse teste, o ritmo de corrida era imposto por sinal acústico emitido por aparelho de reprodução de um suporte audio.
Para a classificação de sobrepeso e de obesidade nos meninos e meninas estudados, foram utilizados como pontos de corte os valores dos percentis $85 \mathrm{e}$ 95 , respectivamente, de acordo com o sugerido por Dietz e Bellizzi(9) e também, os valores de corte internacional sugeridos por Cole et al.(8). Estes últimos pontos de corte(8) para classificar o sobrepeso e a obesidade na infância e adolescência resultam da utilização do método LMS e baseiam-se nos valores de corte de sobrepeso $\left(25 \mathrm{Kg} / \mathrm{m}^{2}\right)$ e obesidade (30 $\mathrm{Kg} / \mathrm{m}^{2}$ ) para a idade adulta, sendo no entanto os valores ajustados para a idade e para o sexo.

\section{Tratamento dos dados}

Para o tratamento e análise dos dados foram utilizadas as medidas habituais da estatística descritiva (média, desvio padrão e frequências) e o teste $t$ independente de Student, $(\mathrm{p} \leq 0,05)$, para as comparações entre sexos nas variáveis idade, massa corporal, estatura, altura tronco-cefálica, altura trocantérica, perímetro da cintura, o perímetro do quadril, dobras cutâneas tricipital e subescapular, IMC, relação cintura quadril, somatório das dobras cutâneas tricipital e subescapular, percentual de gordura, massa gorda, massa corporal magra, flexibilidade, dinamometria pela mão direita e esquerda e consumo máximo de oxigénio.

Para estudar as associações entre o percentual de gordura, IMC, razão cintura-quadril, perímetro da cintura, perímetro do quadril e massa corporal magra, e ainda entre a massa corporal magra e força de preensão manual pelo hemicorpo direito e esquerdo, recorreu-se à correlação linear de Pearson. A análise de regressão múltipla, método stepwise, foi utilizada com o intuito de verificar a variância na variável dependente IMC devida às variáveis independentes percentual de gordura, massa corporal magra e idade.

\section{RESULTADOS}

Os valores médios da idade, massa corporal, estatura, altura tronco-cefálica, perímetro da cintura, perímetro do quadril, dobra cutânea subescapular e IMC foram mais elevados para o sexo masculino mas sem diferença estatisticamente significativa; o mesmo ocorreu para as variáveis relação cintura quadril, massa corporal magra, dinamometria pela mão direi- 
Tabela 1. Valores da idade, das variáveis antropométricas, de indicadores de adiposidade e aptidão física dos meninos e meninas pré-púberes.

\begin{tabular}{|c|c|c|}
\hline Variáveis & Meninos & Meninas \\
\hline Idade (anos) & $8,6 \pm 0,7$ & $8,4 \pm 0,6$ \\
\hline Estatura $(\mathrm{cm})$ & $133,3 \pm 6,7$ & $132,7 \pm 7,0$ \\
\hline Altura tronco-cefálica $(\mathrm{cm})$ & $69,1 \pm 3,6$ & $68,4 \pm 3,7$ \\
\hline Altura trocantérica $(\mathrm{cm})$ & $64,1 \pm 3,6$ & $64,3 \pm 3,9$ \\
\hline Perímetro da cintura $(\mathrm{cm})$ & $60,3 \pm 6,3$ & $58,0 \pm 5,3$ \\
\hline Perímetro do quadril (cm) & $71,2 \pm 7,9$ & $70,7 \pm 7,1$ \\
\hline Dobra cutânea subescapular (mm) & $9,3 \pm 6,8$ & $8,3 \pm 4,2$ \\
\hline Dobra cutânea do tríceps (mm) & $11,6 \pm 5,6$ & $12,7 \pm 4,6$ \\
\hline Massa corporal (Kg) & $30,5 \pm 7,0$ & $29,36 \pm 5,9$ \\
\hline IMC $\left(\mathrm{Kg} / \mathrm{m}^{2}\right)$ & $16,9 \pm 2,7$ & $16,5 \pm 2,5$ \\
\hline Relação cintura quadril * & $0,84 \pm 0,03$ & $0,82 \pm 0,04$ \\
\hline$\sum$ das dobras cutâneas trícipital e subescapular ( $\mathrm{mm}$ ) & $21,0 \pm 11,9$ & $21,0 \pm 8,3$ \\
\hline Percentual de gordura & $17,0 \pm 7,8$ & $19,9 \pm 5,9$ \\
\hline Massa gorda $[\mathrm{Kg}]$ & $5,7 \pm 4,0$ & $6,0 \pm 2,9$ \\
\hline Massa corporal magra $(\mathrm{Kg})^{*}$ & $25,2 \pm 3,5$ & $23,2 \pm 3,7$ \\
\hline Flexibilidade $(\mathrm{cm})$ & $23,9 \pm 4,7$ & $24,6 \pm 4,4$ \\
\hline Dinamometria mão direita $(\mathrm{Kg}) *$ & $16,9 \pm 2,9$ & $15,3 \pm 2,4$ \\
\hline Dinamometria mão esquerda $(\mathrm{Kg})$ * & $16,1 \pm 2,6$ & $14,6 \pm 2,8$ \\
\hline $\mathrm{VO}_{2} \max \left(\mathrm{ml} \mathrm{Kg}^{-1} \cdot \mathrm{min}^{-1}\right)^{*}$ & $47,9 \pm 3,5$ & $45,7 \pm 2,5$ \\
\hline
\end{tabular}

* diferente entre meninos e meninas $(p \leq 0,05)$

ta e esquerda e $\mathrm{VO}_{2 \max }$ mas nestas, foram encontradas diferenças estatisticamente significativas para osexo feminino. Para o sexo feminino os valores médios da altura trocantérica, dobra cutânea do tríceps, o percentual de gordura, massa gorda e flexibilidade foram superiores aos registados no sexo masculino e foi similar o valor médio encontrado para a soma das dobras cutâneas tríceps e subescapular (Tabela 1$)$, todas as variáveis $(p>0,05)$. Foram encontradas diferenças significativas $(p \leq 0,05)$ entre meninos e meninas pré-púberes para relação cinturaquadril, massa corporal magra, dinamometria manual pela mão direita e esquerda e consumo máximo de oxigénio (Tabela 1).
Os meninos e meninas estão classificados abaixo do nível de sobrepeso ou obesidade pelas classificações do IMC(8) e em nível óptimo no referente ao acúmulo de gordura corporal relativa e ou a soma do valor absoluto das dobras cutâneas(19) (ver Tabela 1). Os meninos estão $5,9 \mathrm{ml} \cdot \mathrm{Kg}^{-1} \cdot \mathrm{min}^{-1} \mathrm{e}$ as meninas 5,7 $\mathrm{ml} . \mathrm{Kg}^{-1} \cdot \mathrm{min}^{-1}$ acima da referência em relação a aptidão cardiorespiratória sugerida por Howley e Franks ${ }^{(13)}$. Em relação à flexibilidade os meninos e as meninas estão entre o percentil 25 e 50 pelo referencial da AAHPERD(1). No referente à dinamometria manual os meninos e meninas apresentam as mesmas diferenças de aplicação força entre a mão esquerda e a direita do referencial sugerido por Johnson e 
Tabela 2. Correlação dos indicadores de adiposidade em meninos pré-púberes.

\begin{tabular}{|c|c|c|c|c|c|}
\hline & IMC & RCQ & $\begin{array}{l}\text { Perímetro } \\
\text { da cintura }\end{array}$ & $\begin{array}{l}\text { Perímetro } \\
\text { do quadril }\end{array}$ & $\begin{array}{c}\text { Massa } \\
\text { corporal magra }\end{array}$ \\
\hline$\%$ de gordura & $0,88 *$ & $-0,11$ & $0,89 *$ & $0,88 *$ & $0,62 *$ \\
\hline IMC & & $-0,09$ & $0,93 *$ & $0,92 *$ & $0,76 *$ \\
\hline RCQ & & & 0,06 & $-0,33$ & $-0,16$ \\
\hline Perímetro da cintura & & & & $0,91 *$ & $0,82 *$ \\
\hline Perímetro do quadril & & & & & $0,84 *$ \\
\hline
\end{tabular}

*Correlação significativa $(p<0,05)$

Tabela 3. Correlação dos indicadores de adiposidade em meninas pré-púberes.

\begin{tabular}{|c|c|c|c|c|c|}
\hline & IMC & RCQ & $\begin{array}{l}\text { Perímetro } \\
\text { da cintura }\end{array}$ & $\begin{array}{l}\text { Perímetro } \\
\text { do quadril }\end{array}$ & $\begin{array}{c}\text { Massa } \\
\text { corporal magra }\end{array}$ \\
\hline$\%$ de gordura & 0,76 * & $-0,32$ & $0,58 *$ & $0,70 *$ & 0,29 \\
\hline IMC & & $-0,14$ & $0,85 *$ & $0,84 *$ & $0,66 *$ \\
\hline $\mathrm{RCQ}$ & & & 0,13 & $-0,41 *$ & $-0,20$ \\
\hline Perímetro da cintura & & & & $0,84 *$ & $0,76 *$ \\
\hline Perímetro do quadril & & & & & $0,79 *$ \\
\hline
\end{tabular}

*Correlação significativa $(p<0,05)$

Nelson(16), ou seja, 0,8 $\mathrm{Kg}$ os meninos e 0,7 Kg as meninas e também, ambos os sexos estão acima dos valores de referência sugeridos pelos autores.

Foram encontradas correlações significativas, para os meninos e meninas, entre (i) o percentual de gordura com o IMC, perímetro da cintura e o perímetro do quadril; (ii) entre o IMC com a massa corporal magra, perímetro da cintura e o do quadril; (iii) o perímetro da cintura com o perímetro do quadril e massa corporal magra e (iv) o perímetro do quadril e a massa corporal magra. Em ambos os sexos, não foram encontradas correlações significativas entre a razão cintura quadril (RCQ) com o percentual de gordura, entre o IMC e o perímetro da cintura e entre a massa corporal magra com a relação cintura quadril. É importante ressaltar que a correlação da RCQ com o IMC, percentual de gordura, perímetro do quadril e massa corporal magra foram negativas nos meninos e meninas estudados. Encontrou-se correlação significativa para o sexo masculino entre massa corporal magra com o percentual de gordura, enquanto para o sexo feminino essa mesma correlação não foi significativa. No sexo feminino a correlação entre perímetro do quadril com razão cintura quadril foi significativa mas não no sexo masculino (Tabelas 3 e 4). Portanto, nas crianças pré-púberes, existe correlação significativa entre os indicadores de adiposidade corporal estudados (IMC, percentual de gordura, perímetro da cintura e perímetro do quadril), excepto com a razão cintura quadril.

Nos percentis 85 e 95 foram mais elevados para os meninos os valores médios das variáveis IMC, percentual de gordura, somatório das dobras cutâneas tricipital e subescapular, razão cintura quadril, perímetros da cintura e do quadril (Tabela 5 e 6). Em ambos os sexos, os sujeitos estudados foram classificados em sobrepeso quando se encontravam entre os percentis 85 e 95 IMC e como obesos quando estavam no percentil 95 ou acima deste ${ }^{(8)}$.

No sexo masculino, os valores absolutos e relativos da 
Tabela 4. Valores médios dos indicadores de adiposidade de meninos pré-púberes de acordo com valores do percentil.

\begin{tabular}{lcc}
\hline Variáveis & Percentil 85 & Percentil 95 \\
\hline IMC $\left(\mathrm{Kg} / \mathrm{m}^{2}\right)$ & 19,4 & 23,8 \\
\hline Percentual de gordura & 28,4 & 33,1 \\
\hline$\sum$ das dobras cutâneas tríceps e subescapular $(\mathrm{mm})$ & 35,8 & 50,5 \\
\hline Relação cintura quadril & 0,88 & 0,93 \\
\hline Perímetro da cintura $(\mathrm{cm})$ & 67,5 & 75,2 \\
\hline Perímetro do quadril $(\mathrm{cm})$ & 79,5 & 90,5 \\
\hline
\end{tabular}

Tabela 5. Valores médios dos indicadores de adiposidade de meninas pré-púberes de acordo com valores do percentil.

\begin{tabular}{lcc}
\hline Variáveis & Percentil 85 & Percentil 95 \\
\hline $\mathrm{IMC}\left(\mathrm{Kg} / \mathrm{m}^{2}\right)$ & 19,0 & 22,2 \\
\hline Percentual de gordura & 26,1 & 32,4 \\
\hline$\sum$ das dobras cutâneas tríceps e subescapular $(\mathrm{mm})$ & 28,2 & 40,6 \\
\hline Relação cintura quadril & 0,86 & 0,90 \\
\hline Perímetro da cintura $(\mathrm{cm})$ & 64,4 & 68,4 \\
\hline Perímetro do quadril $(\mathrm{cm})$ & 79,2 & 86,0 \\
\hline
\end{tabular}

gordura corporal, estão classificados como altos no percentil 85 e muito altos no percentil 95 . No sexo feminino, quando se consideram os valores da gordura corporal relativa, estes estão classificados como moderadamente altos no percentil 85 e altos no percentil 95, enquanto pela soma das dobras cutâneas são classificados em moderadamente altos e muito altos, nos percentis 85 e 95, respectivamente(19). A variância do IMC é explicada pelo percentual de gordura em $78,2 \%$ nos meninos e em $58,6 \%$ nas meninas enquanto a massa corporal magra explica a variância do IMC em 59\% e 44,3\%, respectivamente no sexo masculino e feminino e o percentual de gordura e a massa corporal magra em $85,8 \%$ e $79,5 \%$. Considerando adicionalmente a variável idade, foi possível verificar melhor predição da variável dependente IMC pelos preditores analisados. Deste modo, o percentual de gordura e a idade explicam o IMC de 81,1 (meninos) e $62,3 \%$ (meninas), em $64,2 \%$ e $51,8 \%$ pela massa corporal magra e idade e $89,7 \%$ em ambos os sexos, pelo percentual de gordura, pela massa corporal magra e pela idade (Tabela 6).

\section{DISCUSSÃO}

Utilizando-se como referência o percentil 50 (P50) foi possível verificar que as crianças envolvidas no presente estudo, meninos e meninas, apresentaram os valores de 28,8 e $31,0 \mathrm{Kg}$ para a massa corporal, 134,2 e $136,3 \mathrm{~cm}$ para a estatura e de 16,31 e 15,9 $\mathrm{Kg} / \mathrm{m}^{2}$ para o IMC, respectivamente. Em estudo realizado em amostra da população de Pernambuco e de Sergipe foi possível verificar que o valor do P50 foi de 26,7 e $27,5 \mathrm{Kg}$ (meninos) e de 24,5 e $26,4 \mathrm{Kg}$ (meninas) para a massa corporal, 129,9 e 133,1 cm e de 129,4 e $131,6 \mathrm{~cm}$ para a estatura e 16,1 e 15,9 $\mathrm{Kg} / \mathrm{m}^{2}$ e de 15,4 e de $15,8 \mathrm{Kg} / \mathrm{m}^{2}$ para o IMC, respectivamente(23). Deste modo, verificou-se que os meninos e meninas agora estudados em Curitiba apresentam valores mais elevados para a massa corporal e estatura. Em relação ao IMC o valor para 
Tabela 6. Equações obtidas na regressão para estimar o IMC e variação no IMC devido aos preditores percentual de gordura, massa corporal magra e idade.

\begin{tabular}{|c|c|c|}
\hline Variáveis independentes & Meninos & Meninas \\
\hline Percentual de gordura $(\% G)$ & $\begin{array}{c}\mathrm{IMC}=11,796+(0,314 \times \% \mathrm{G}) \\
\mathrm{SEE}=1,33 ; \mathrm{R} 2=78,2 \%\end{array}$ & $\begin{array}{c}\mathrm{IMC}=9,955+(0,331 \times \% \mathrm{G}) \\
\mathrm{SEE}=1,66 ; \mathrm{R} 2=58,6 \%\end{array}$ \\
\hline $\begin{array}{l}\text { Percentual de Gordura }(\% G \text { ] } \\
\text { e idade (anos) }\end{array}$ & $\begin{array}{c}\mathrm{IMC}=17,636+(0,313 \times \% \mathrm{G}) \\
-(0,673 \times \mathrm{IDADE}) \\
\mathrm{SEE}=1,26 ; \mathrm{R} 2=81,1 \%\end{array}$ & $\begin{array}{c}\text { IMC }=16,087+(0,344 \times \% G) \\
-(0,753 \times \text { IDADE }) \\
\text { SEE }=1,61 ; \mathrm{R} 2=62,3 \%\end{array}$ \\
\hline Massa corporal magra (MCM, Kg) & $\begin{array}{l}\mathrm{IMC}=1,738+(0,611 \times \mathrm{MCM}) \\
\quad \mathrm{SEE}=1,82 ; \mathrm{R} 2=59,0 \%\end{array}$ & $\begin{array}{c}\mathrm{IMC}=6,041+(0,451 \times \mathrm{MCM}) \\
\quad \mathrm{SEE}=1,93 ; \mathrm{R} 2=44,3 \%\end{array}$ \\
\hline $\begin{array}{l}\text { Massa corporal magra (MCM, Kg) } \\
\text { e Idade (anos) }\end{array}$ & $\begin{array}{c}\mathrm{IMC}=9,267+(0,623 \times \mathrm{MCM}) \\
-(0,904 \times \mathrm{IDADE}) \\
\mathrm{SEE}=1,73 ; \mathrm{R} 2=64,2 \%\end{array}$ & $\begin{array}{c}\text { IMC }=14,158+(0,507 \times \mathrm{MCM}) \\
-(1,109 \times \mathrm{IDADE}) \\
\mathrm{SEE}=1,82 ; \mathrm{R} 2=51,8 \%\end{array}$ \\
\hline $\begin{array}{l}\text { Percentual de gordura e Massa } \\
\text { corporal magra (MCM, Kg) }\end{array}$ & $\begin{array}{c}\mathrm{IMC}=6,045+(0,235 \times \% \mathrm{G}) \\
+(0,281 \times \mathrm{MCM}) \\
\mathrm{SEE}=1,09 ; \mathrm{R} 2=85,8 \%\end{array}$ & $\begin{array}{c}\mathrm{IMC}=3,621+(0,269 \times \% \mathrm{G}) \\
+(0,325 \times \mathrm{MCM}) \\
\mathrm{SEE}=1,19 ; \mathrm{R} 2=79,5 \%\end{array}$ \\
\hline $\begin{array}{l}\text { Percentual de gordura, Massa } \\
\text { corporal magra (MCM, Kg) e Idade (anos) }\end{array}$ & $\begin{array}{c}I M C=12,422+(0,230 \times \% G) \\
+(0,299 \times M C M)-(0,778 \times \text { IDADE }) \\
\text { SEE = 0,95; R2 }=89,7 \%\end{array}$ & $\begin{array}{c}I M C=12,995+(0,280 \times \% G) \\
+(0,385 \times M C M)-(1,294 \times \text { IDADE }) ; \\
\text { SEE }=0,85 ; R 2=89,7 \%\end{array}$ \\
\hline
\end{tabular}

SEE = erro padrão de estimativa.

ambos os sexos em Curitiba foi discretamente mais elevado do que em Pernambuco e Sergipe.

Em crianças pré-púberes na cidade de Porto Alegre, os valores médios encontrados em meninos e meninas para a massa corporal foram, respectivamente, de $33,8 \mathrm{Kg}$ e $29,9 \mathrm{Kg}$, de $135,3 \mathrm{~cm}$ e $133,0 \mathrm{~cm}$ para a estatura, de $18,2 \mathrm{Kg} / \mathrm{m}^{2}$ e $16,8 \mathrm{Kg} / \mathrm{m}^{2}$ para o IMC e para o somatório de dobras cutâneas tricipital e subescapular de 21,3 mm e 23,2 $\mathrm{mm}^{(22)}$. Utilizando a mesma equação do presente estudo para o cálculo do percentual de gordura, da massa gorda e da massa corporal magra foi possível verificar que os meninos e meninas pré-púberes de Porto Alegre(22) apresentaram valores de $18,9 \%$ e $22,4 \%$ gordura, $6,3 \mathrm{Kg}$ e $6,7 \mathrm{Kg}$ de massa gorda e $27,4 \mathrm{Kg}$ e $23,1 \mathrm{Kg}$ massa corporal magra. No estudo realizado em Porto Alegre os valores encontrados foram mais elevados para os meninos nas variáveis massa corporal, estatura, IMC, \% gordura e massa corporal magra e discretamente mais elevado o valor para o somatório das dobras cutâneas e massa gorda; para as meninas discretamente mais elevados foram os valores da massa corporal, estatura, IMC e massa gorda e mais elevados os valores do somatório das dobras cutâneas e percentual de gordura e discretamente menos elevado o valor da massa corporal magra. Tanto no estudo realizado em Porto Alegre(22) quanto no presente realizado em Curitiba, foram mais elevados para os meninos os valores da massa corporal, estatura, IMC e massa corporal magra e nas meninas o $\%$ gordura e massa gorda. A única divergência encontrada entre os resultados do presente estudo e o de Porto Alegre(22) foi em relação ao somatório das dobras cutâneas, pois, foi similar entre sexos prépúberes em Curitiba e mais elevado para as meninas em Porto Alegre.

Na pré-adolescência a proporção do tecido adiposo e a da massa corporal magra em meninos e meninas são similares (28). Esta colocação reforça os resultados deste estudo em relação à não existência de diferenças significativas entre sexos para a massa corporal, estatura, IMC, percentual de gordura e os perímetros da cintura e do quadril, ou seja, retrata a similaridade entre meninos e meninas pré-púberes dos indicado- 
res de adiposidade e tamanho corporal. Não foi similar entre sexos a proporção da massa corporal magra e este resultado discorda dos achados de Wang(28). Os meninos pré-púberes neste estudo realizado em Curitiba apresentaram 2,0 Kg a mais de massa corporal magra; e em Porto Alegre 4,1 Kg a mais de massa corporal magra do que as meninas(22) e, isto, retrata similaridade em termos dos meninos pré-púberes apresentarem maior quantidade de massa corporal magra do que as meninas. Para os meninos que não são maduros sexualmente ocorre o aumento da massa corporal magra e do percentual de gordura devido ao efeito da testosterona ${ }^{(4)}$. Esta colocação pode explicar o motivo de nos estudos os meninos apresentarem quantidade mais elevada de massa corporal magra do que as meninas e, também, a correlação significativa encontrada para os meninos entre percentual de gordura e massa corporal magra. Os meninos apresentaram mais força do que as meninas no teste de força isométrica(22) e este resultado está de acordo com achados do presente estudo. O sexo masculino apresentou valores mais elevados para a massa corporal magra e também para a força de preensão manual realizada pelo hemicorpo direito e esquerdo do que o sexo feminino, $(\mathrm{p}<0,05)$. Este resultado pode explicar a diferença de força entre sexos, pois, existiu correlação significativa e positiva entre massa corporal magra e força de preensão manual para o sexo masculino e feminino. Deste modo, provavelmente, o sexo masculino apresentou valores mais elevados nos testes de força de preensão manual pelo hemicorpo direito e esquerdo devido a possuir valor mais elevado em relação à massa corporal magra.

As meninas apresentaram valores de flexibilidade mais elevados do que os meninos e este resultado está em discordância com os resultados do estudo de Christodoulos et al.(7), pois, os meninos apresentaram $1,1 \mathrm{~cm}$ a mais de flexibilidade do que as meninas. No presente estudo e nos dois sexos, foram observados valores mais elevados no teste de flexibilidade do que nos meninos e meninas estudados na Grécia, sendo a diferença de $8,1 \mathrm{~cm}$ no sexo masculino e $9,9 \mathrm{~cm}$ no sexo feminino.

Os valores do $\mathrm{VO}_{2 \max }$ relativo estimado nos meninos e meninas do presente estudo foi superior ao valor encontrado em estudo realizado na Grécia (7) em
24,1 e $22,4 \mathrm{ml} . \mathrm{Kg}^{-1} \cdot \mathrm{min}^{-1}$, respectivamente..A semelhança entre este estudo e aquele anteriormente referido residiu apenas no facto de os meninos apresentam valores mais elevados do $\mathrm{VO}_{2 \max }$ do que as meninas. Deste modo, neste estudo o $\mathrm{VO}_{2 \max }$ relativo das meninas correspondeu a $95,4 \%$ do $\mathrm{VO}_{2 \max }$ relativo dos meninos e na Grécia o das meninas correspondeu a $97,8 \%$ do $\mathrm{VO}_{2 \max }$ relativo dos meninos. No presente estudo, observou-se correlação significativa entre os indicadores de adiposidade e da composição corporal (IMC, percentual de gordura, perímetro da cintura e perímetro do quadril) das crianças pré-púberes estudadas, excepto com a razão cintura quadril . Em estudo efectuado por Teixeira et al.(26) não se registaram correlações significativas entre a razão cintura quadril e as variáveis IMC, \% gordura, massa gorda, somatório de dobras cutâneas do tronco ou da extremidade e esses resultados estão em conformidade com os deste. O que neste estudo de Curitiba está em conformidade com o estudo de Taylor et al.(25) é o fato de que o percentual de gordura se correlacionar de forma elevada com o IMC em crianças e adolescentes do sexo masculino $(r>0,84)$ e feminino $(r>0,83)$. No estudo de Taylor et al.(25) a correlação entre gordura localizada no tronco e o perímetro da cintura foi significativamente mais elevada em crianças e adolescentes do sexo masculino e feminino $(r=0,92, p<0,01)$ do que a correlação entre gordura localizada no tronco e relação cintura quadril $(r=-0,40$ e $r=-0,04$, meninos e meninas). Os resultados desse estudo são similares com o nosso, realizado em Curitiba, pois, a correlação entre gordura localizada no tronco e perímetro da cintura foi significativa $(r=0,86$ para os meninos e $\mathrm{r}=0,75$ para as meninas; $\mathrm{p}<0,01$ ) e, também, mais elevada do que a correlação entre gordura localizada no tronco e relação cintura quadril $(r=-0,00$ para os meninos e $r=-0,16$ para as meninas; $p>0,05$. Estes resultados sugerem que o perímetro da cintura representa melhor a gordura localizada no tronco do que a razão cintura quadril. Interessante ressaltar que os perímetros da cintura e do quadril apresentaram correlação significativa com a massa corporal magra e com o percentual de gordura e isto indica que os mesmos representaram de forma significativa a massa corporal magra e a gordura corporal relativa 
nos dois sexos. Estas associações significativas, tanto com o percentual de gordura quanto com a massa corporal magra em ambos os sexos, podem ser justificadas pelo estadio maturacional no qual as crianças se enquadram, pois, ainda não ocorreu o início da puberdade, onde nos meninos e por razões hormonais deveria ocorrer um aumento mais acentuado da massa corporal magra do que de massa de gordura e nas meninas ocorrer o inverso, ou seja, um aumento mais elevado da massa gorda(28). Isto explicaria nos meninos a correlação entre os perímetros com constituintes da composição corporal ser entre 0,82 a 0,89 e nas meninas, ser entre 0,58 a 0,79 , com a característica dos perímetros da cintura e quadril representarem melhor a variação na massa corporal magra do que a no percentual de gordura.

O IMC foi melhor predito no sexo masculino e no feminino pelas variáveis independentes massa corporal magra, percentual de gordura e idade do que quando estas variáveis preditoras foram consideradas separadamente ou quando houve a redução de uma destas variáveis. Os resultados vêm a confirmar que o IMC representa o percentual de gordura e a massa corporal magra em crianças e adolescentes de ambos os sexos e varia conforme a idade ${ }^{(18)}$ e, deste modo, pode ser utilizado para estimativa do percentual de gordura corporal e da massa corporal magra, principalmente, quando a variável idade é considerada. O facto do erro padrão de estimativa do percentual de gordura pelo IMC ser de 3,7\% $\left(\mathrm{R}^{2}=78,2 \%\right)$ para os meninos e de $3,8 \%\left(\mathrm{R}^{2}=58,6 \%\right)$ para as meninas justifica a utilização do IMC como um índice válido para estimar a quantidade de gordura corporal no presente estudo, pois, o $\operatorname{ACSM}^{(2)}$ menciona que quando o erro padrão de estimativa do percentual de gordura pelo IMC for superior a 5,0\% deve ser utilizado outro índice para predizer o estado de gordura corporal. Partindo do princípio que o IMC representou $59,0 \%$ nos sexo masculino e $44,3 \%$ no feminino de variância na massa corporal magra, ambos $\mathrm{R}^{2}$, foi possível verificar que o IMC representou melhor a variação no percentual de gordura do que na massa corporal magra.

No Brasil $13,1 \%$ dos meninos e $14,8 \%$ das meninas estão em sobrepeso, nos EUA 25,0\% e 26,3 \%(27) e neste estudo realizado em Curitiba 18,8\% e
$14,3 \%(8)$, respectivamente. Deste modo, o percentual dos Curitibanos em sobrepeso foi mais elevado do que o encontrado para o Brasil e menos elevado do que o para os EUA e o percentual das Curitibanas em sobrepeso é muito próximo do encontrado para o Brasil e inferior ao para os EUA. Estão classificados em sobrepeso $18,8 \%$ dos meninos e $14,3 \%$ das meninas e em obesidade ${ }^{(8)} 6,3$ e $2,9 \%$, respectivamente; em risco de sobrepeso e sobrepeso(6) 15,6 dos meninos e $14,3 \%$ das meninas ou em sobrepeso e obesidade(9) $3,13 \%$ e $2,9 \%$, meninos e meninas, respectivamente,. Portanto, parece existir proximidade para detecção do sobrepeso e obesidade nos meninos e meninas estudados entre os valores médios sugeridos por Cole et al.(8) e Dietz e Bellizzi(9), principalmente, devido aos sexos estudados serem classificados no percentil 85 em sobrepeso e no percentil 95 em obesidade pelo referencial de Cole et al.(8). Também, neste estudo, pelas análises dos valores médios, os meninos e meninas foram classificados abaixo do nível de sobrepeso ou obesidade pelas classificações do IMC(8) e no nível óptimo em relação ao acumulo de gordura corporal relativa e ou a soma do valor absoluto das dobras cutâneas(19), o que indica proximidade entre as classificações sugeridas para os indicadores de adiposidade. Em suma, foi similar a proporção do tecido adiposo e não foi similar a proporção de massa corporal magra entre crianças pré-púberes. Os meninos apresentaram melhor índice de força de preensão manual pela mão direita e esquerda e consumo máximo de oxigénio e as meninas flexibilidade. Existe correlação significativa entre IMC, percentual de gordura e perímetros da cintura e quadril nos sexos. O IMC foi melhor predito no sexo masculino e no feminino pela massa corporal magra, percentual de gordura e idade. O IMC foi considerado um índice válido para predizer o estado de gordura corporal e representou melhor a variância na gordura corporal do que na massa corporal magra. Existiu proximidade para detecção do sobrepeso e obesidade nos meninos e meninas estudados entre os valores médios sugeridos por Cole et al.(6) e Dietz e Bellizzi(9). Portanto, recomenda-se que o sexo e a idade sejam considerados quando se pretende analisar os indicadores de sobrepeso, de obesidade e de aptidão física em crianças pré-púberes. 


\section{CONCLUSÃO}

As variáveis idade, massa corporal, estatura, massa gorda, flexibilidade e as que representam os indicadores de adiposidade corporal (percentual de gordura, IMC, somatório das dobras cutâneas tríceps e subescapular, perímetro da cintura e o do quadril) são homogéneas entre os meninos e as meninas prépúberes estudados. Não são homogéneos os indicadores de aptidão física, massa corporal magra, força de preensão manual e $\mathrm{VO}_{2 \max }$.

Houve correlação significativa em ambos os sexos para o percentual de gordura, IMC, perímetro da cintura e o do quadril para essa população pré-púbere, excepto para a relação cintura quadril. O IMC foi predito de forma mais significativa pela gordura corporal relativa, massa corporal magra e idade; foi considerado um índice válido para predizer o estado de gordura corporal e representou melhor a variância na gordura corporal do que na massa corporal magra. Existe proximidade para detecção do sobrepeso e obesidade nos meninos e meninas estudados entre os valores médios sugeridos por Cole et al.(8) e Dietz e Bellizzi(9).

Recomenda-se considerar o sexo e a idade quando se pretende analisar os indicadores de sobrepeso, de obesidade e de aptidão física em crianças pré-púberes.

\section{CORRESPONDÊNCIA}

\section{Fabrício Barbosa Alves}

Rua Paraguai, 406 - Sobrado 01

82510-090

Bacacheri, Curitiba - PR

E-mail: fabriciobalves@yahoo.com 


\section{REFERÊNCIAS BIBLIOGRÁFICAS}

1. AAHPERD - American Alliance for Health, Physical Education, Recreation and Dance (1988). Physical Best - A Physical Fitness Education \& Assessment Program. Reston, Virginia: AAHPERD.

2. ACSM - American College of Sports Medicine (2006). ACSM's Guidelines for Exercise Testing and Prescription. United States: Lippincott Williams \& Wilkins.

3. Boileau RA, Lohman TG, Slaughter MH, Ball TE, Going SB, Hendrix MK (1984). Hydration of the fat-free body in children during maturation. Hum Biol 56 (6): 651-666

4. Bray GA, Delany JP, Harsha DW, Volaufova J, Champagne CM (2001). Body composition of African American and White Children: A 2-Year Follow-Up of the BAROC Study. Obes Res 9 (11): 605-621.

5. Callaway CW, Chumlea WC, Bouchard C, Himes JH, Lohman TG, Martin AD, Mitchell CD, Mueller WH, Roche AF, Seefeldt VD (1991). CIRCUMFERENCES. In: Lohman TG, Roche AF, Martorell R (eds.). Anthropometric standardization reference manual. United States: Human Kinetics, 3954.

6. CDC - Centers for Disease Control and Prevention. BMI for Children and Teens. Disponível em: <www.cdc.gov> . Acessado em: Janeiro de 2005.

7. Christodoulos AD, Flouris AD, Tokmakidis PS (2006). Obesity and physical fitness of pre-adolescent children during the academic year and the summer period: effect of organized physical activity. J Child Health Care 10: 199211.

8. Cole TJ, Bellizzi MC, Flegal KM, Dietz WH (2000). Establishing a standard difinition for child overweight and obesity worlwide: international survey. BM J 320: 1-6.

9. Dietz WH, Bellizzi MC (1999). Introduction: the use of body mass index to assess obesity in children. Am Soc Clin Nutr, 70: 123-125.

10. Faulkner RA (1996). Maturation. In: Docherty D (ed). Measurement in pediatric exercise science. Canada: Human Kinetics, 129-158.

11. Gordon CC, Chumlea WC, Roche AF (1988). Stature, Recumbent Length, and Weight. In: Lohman TG, Roche AF, Martorell R (eds.). Anthropometric standardization reference manual. United States: Human Kinetics, 03-08.

12. Harrison GG, Buskirk ER, Carter JEL, Johnston FE, Lohman TG, Pollock ML, Roche AF, Wilmore J (1988). Skinfold thicknesses and measurement technique. In: Lohman TG, Roche AF, Martorell R (eds.). Anthropometric standardization reference manual. United States: Human Kinetics, 55-80.

13. Howley ET, Franks DB (1997). Health Fitness Instructor's Handbook. 3rd ed. United States: Human Kinetics.

14. Huang Y, Malina RM (2007). BMI and Health-Related Physical Fitness in Taiwanese Youth 9-18 Years. Med Sci Sports Exerc 39 (5): 701-708.

15. Janssen I, Katzmarzyk PT, Ross R (2004). Waist circumference and not body mass index explains obesity-related health risk. Am J Clin Nutr 79: 379-384.

16. Johnson BR, Nelson JK (1986). Pratical Measurements for evaluation in physical education. 4th ed.. United States: Burgess Publishing.

17. Léger LA, Mercier D, Gadaoury C, Lambert J (1988). The multistage 20 metre shuttle run test for aerobic fitness. J Sports Sci 6: 93-101.
18. Lindsay RS, Hanson RL, Roumain J, Ravussin E, Knowler WC, Tataranni PA (2001). Body Mass Index as a Measure of Adiposity in Children and Adolescents: Relationship to Adiposity by Dual Energy X-Ray Absorptiometry and to Cardiovascular Risk Factors. J Clin Endocrionol Metab 86 (10): 4061-4067.

19. Lohman TG (1992). Advances in body composition assessment. United States: Human Kinetics Publishers.

20. Mccarthy HD, Ellis SM, Cole TJ (2003). Central overweight and obesity in British youth aged 11-16 years: cross sectional surveys of waist circumference. BMJ 326: 624-628.

21. Safrit MJ (1986). Introduction to measurement in Physical Education and Exercise Science. St. Louis: Times Mirror/Mosby College Publishing.

22. Schneider P, Rodrigues LA, Meyer F (2002). Dinamometria computadorizada como metodologia de avaliação da força muscular de meninos e meninas em diferentes estágios de maturidade. Rev Paul Educ Fís 12 (1): 35-42.

23. Silva RJS, Júnior AGS, Oliveira ACC (2005). Crescimento em crianças e adolescentes: Um estudo comparativo. Rev Bras Cine Des Hum 7 (1): 12-20.

24. Sun SS, Schubert CM, Chumlea WC, Roche AF, Kulin HE, Lee PA, Himes JH, Ryan AS (2002). National Estimates of the Timing of Sexual Maturation and Racial Differences Among US Children. Pediatrics 110 (6): 911-919.

25. Taylor RW, Jones IE, Williams SM, Goulding A (2000). Evaluation of waist circumference, waist-to-rip ratio, and the conicity index as screening tools for high trunk fat mass, as measured by dual-energy X-ray absorptiometry, in children aged 3-19 y-3. Am J Clin Nutr 72: 490-495.

26. Teixeira PJ, Sardinha LB, Going SB, Lohman TG (2001). Total and Regional Fat and Serum Cardiovascular Disease Risk Factors in Lean and Obese Children and Adolescents. Obes Res 9 (9): 432-442.

27. Wang W, Monteiro C, Popkin BM (2002). Trends of obesity and underweight in older children and adolescents in the United States, Brazil, China, and Russia. Am J Clin Nut 75: 971-977.

28. Wang Y (2002). Is obesity associated with early sexual maturation? A comparison of the association in American boys versus girls. Pediatrics 110 (6): 903-910.

29. Whitlock EP, Williams SB, Gold R, Smith PR, Shipman SA (2005). Screening and Interventions for Childhood Overweight: A Summary of Evidence for the US Preventive Services Task Force. Pediatrics 116 (1): e125-144.

30. Wilson DM, Wang Y, Cullen KW, Baranowski T, Himes JH, Mcclanahan BS, Robinson TN (2004). Assessing WeightRelated Biochemical Cardiovascular Risk Factors in African American Girls. Obes Res 12: 73-83. 